\title{
Thermal storage and insulation properties of a diver dry suit
}

\author{
M. F. Ding ${ }^{1} \quad$ J. A. Gear ${ }^{2} \quad$ Y. Ding ${ }^{3}$
}

(Received 29 May 2010; revised 16 August 2010)

\begin{abstract}
Thermal storage and insulation properties of a diver dry suit incorporating phase change material are investigated. A complete model for the fabric, air-gap, skin system is developed. The numerical results show that the diver dry suit consisting of phase change material and conventional insulating materials provides significantly enhanced thermal protection under cold environmental conditions. A sensitivity analysis investigates how changes in the values of the thickness of air space affect the temperature distribution in the system.
\end{abstract}

\section{Contents}

1 Introduction

C626

2 The mathematical model

C627

http://anziamj . austms.org.au/ojs/index.php/ANZIAMJ/article/view/2545 gives this article, (C) Austral. Mathematical Soc. 2010. Published August 25, 2010. ISSN 1446-8735. (Print two pages per sheet of paper.) Copies of this article must not be made otherwise available on the internet; instead link directly to this URL for this article. 
2.1 Heat transfer model in fabric and air layer . . . . . . . . . C627

2.2 Heat transfer model in skin . . . . . . . . . . . . . . C630

2.3 Boundary and initial conditions . . . . . . . . . . C630

2.4 Numerical method .. . . . . . . . . . . . . C633

3 Results and discussions

C634

References

C638

\section{Introduction}

A phase change material (PCM) is a type of polymer substance with heatresistant capacities encapsulated in either microscopic or macroscopic balls. When a PCM reaches its melting point or crystallisation point it absorbs or releases large amounts of heat with small temperature variation.

Due to the high latent heat absorption or release that occurs upon the change of phase, an encapsulated PCM incorporated within textile fabric enhances the thermal protection during either hot or cold environmental conditions [3, 4, 7]. The use of PCM in clothing dates from the 1980s [4].

Nuckols [7] developed an analytical model to assess the thermal protection provided divers with PCM-enhanced dry suits. On the basis of Nuckols' work, Gear et al. [3] investigated the thermal storage and insulation properties of a multi-layered diver dry suit containing PCM. The model assumed a constant temperature at the boundary that was in contact with the skin. This assumption implies an infinite energy supply to the PCM-enhanced fabric. A bio-heat transfer model that incorporates heat flow through human skin is required.

This article reports the development of the bio-heat transfer model for evaluating the thermal performance of a diver dry suit enhanced with PCM. The model analyzes the heat transfer through human skin, an air-gap and a two 
layer PCM-enhanced fabric. A finite difference scheme was used to obtain the numerical solution of the temperature distribution inside the fabric, air-gap, skin system. For comparison, we also calculate the temperature distribution in a system where no PCM is incorporated within the dry suit.

\section{The mathematical model}

As shown in Figure 1, the geometry of the model consists of PCM-enhanced fabric, air-gap and skin. The PCM-enhanced fabric is composed of two layers, which are constructed from a micro-fibrous insulating material called Thinsulate $^{1}$ (outer layer) and Comfortemp foam enhanced with phase change material, C18 paraffin (octadecane), encapsulated in Thermosorb,$^{2}$ see formulation $B$ by Nuckols [7]. These two layers are denoted by $R_{1}$ and $R_{2}$.

In accordance with other skin models $[1,6,8,9]$, the skin is assumed to consist of three layers with different physiological properties: the epidermis (outermost layer of skin), the dermis (intermediate layer), and the subcutaneous fatty layer. These three layers are denoted by $R_{4}, R_{5}$ and $R_{6}$. An air-gap $\left(R_{3}\right)$ lies between fabric and skin. Due to the large surface area of each layer of the fabric, air-gap, skin system and its small thickness, a mathematical model for each layer is expressed as one-dimensional heat flow in a continuum.

\subsection{Heat transfer model in fabric and air layer}

The following standard one dimensional heat diffusion equation is used to describe the heat transfer in Thinsulate and air layers.

$$
\rho c \frac{\partial T}{\partial t}=\frac{\partial}{\partial x}\left(k \frac{\partial T}{\partial x}\right) \quad \text { in } R_{i} \text { for } i=1,3 .
$$

${ }^{1}$ Thinsulate is a trademark of the $3 \mathrm{M}$ Corporation

${ }^{2}$ Comfortemp and Thermosorb are trade names used by Frisby Technologies of Clemmons, NC. 


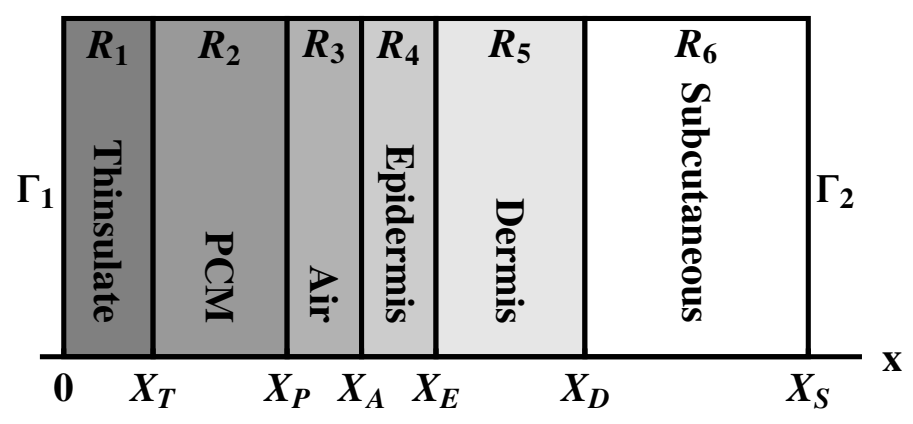

FiguRE 1: Schematic diagram of the model geometry.

Here $\mathrm{T}$ is temperature at time $t, k$ is the thermal conductivity, $\rho$ is the density and $\mathrm{c}$ is the specific heat.

For phase change problems the situation is slightly more complicated because of latent heat. For the phase change material considered here, the phase change occurs over a temperature range of about $1{ }^{\circ} \mathrm{C}$ and the thermal conductivities and specific heats vary only slightly with temperature over the range of temperatures considered $\left(4-37^{\circ} \mathrm{C}\right)$. The most significant feature which determines the thermal protection, or temperature control properties of the fabric, is the latent heat $L\left[\mathrm{~J}_{\mathrm{kg}}{ }^{-1}\right]$ (the jump in enthalpy at the phase change is $\rho \mathrm{L}$ ). The density in the phase change layer is almost completely dominated by Comfortemp foam which is relatively massive compared to micro-encapsulated PCM. Hence the density in the phase change layer is taken as constant [7]. Let $T_{f}$ be the fusion temperature and $c_{s}$ and $c_{l}$ be constant specific heats in the solid and liquid phases. The enthalpy $H(T)$ is defined by [5]

$$
H(T)= \begin{cases}\rho c_{s} T, & T<T_{f}, \\ {\left[\rho c_{s} T_{f}, \rho\left(c_{s} T_{f}+L\right)\right],} & T=T_{f}, \\ \rho\left(L+\left(c_{s}-c_{l}\right) T_{f}\right)+\rho c_{l} T, & T>T_{f},\end{cases}
$$

where $[a, b]$ denotes that $H(T)$ takes any value in the interval. The heat 
TABLE 1: Thermophysical/geometrical properties of the fabric [3].

\begin{tabular}{cccc}
\hline Property & PCM solid & PCM liquid & Thinsulate \\
\hline $\mathrm{c}\left[\mathrm{J} / \mathrm{kg} /{ }^{\circ} \mathrm{K}\right]$ & 1483 & 1620 & 1129.68 \\
$\mathrm{k}\left[\mathrm{W} / \mathrm{m} /{ }^{\circ} \mathrm{K}\right]$ & 0.03478 & 0.03798 & 0.03286 \\
Width $[\mathrm{mm}]$ & \multicolumn{2}{c}{7.62} & 5.08 \\
$\rho\left[\mathrm{kg} / \mathrm{m}^{3}\right]$ & 121.43 & 52.86 \\
$\mathrm{~L}[\mathrm{~J} / \mathrm{kg}]$ & 99424.87 & \\
$\mathrm{~T}_{\mathrm{f}}\left[{ }^{\circ} \mathrm{C}\right]$ & 28.3333 & \\
\hline
\end{tabular}

TABLE 2: Thermophysical/geometrical properties of the air-layer and human skin $[1,6]$.

\begin{tabular}{cccccc}
\hline Property & Air-gap & Epidermis & Dermis & Subcutaneous & Blood \\
\hline$\rho\left[\mathrm{kg} / \mathrm{m}^{3}\right]$ & 1.18 & 1200 & 1200 & 1000 & 1100 \\
$\mathrm{c}\left[\mathrm{J} / \mathrm{kg} /{ }^{\circ} \mathrm{K}\right]$ & 1005 & 3600 & 3300 & 2500 & 3770 \\
$\mathrm{k}\left[\mathrm{W} / \mathrm{m} /{ }^{\circ} \mathrm{K}\right]$ & 0.026 & 0.21 & 0.37 & 0.16 & \\
$\mathrm{Width}[\mathrm{mm}]$ & $0.3-1$ & 0.080 & 2.0 & 10.0 & \\
$\omega\left[\mathrm{m}^{3} / \mathrm{s} / \mathrm{m}^{3}\right]$ & & 0 & $0-0.000381$ & 0.000381 & \\
$\mathrm{~S}\left[\mathrm{~W} / \mathrm{m}^{3}\right]$ & & 0 & $0-3684.38$ & 3684.38 & \\
\hline
\end{tabular}

diffusion equation in the phase change layer is $[3,5]$

$$
\frac{\partial H}{\partial t}=\frac{\partial}{\partial x}\left(k(T) \frac{\partial T}{\partial x}\right) \quad \text { in } R_{2} .
$$

The thermal conductivity $k(T)$ is constant in each phase; that is,

$$
k(T)= \begin{cases}k_{s}, & T<T_{f}, \\ k_{l}, & T>T_{f},\end{cases}
$$

where $k_{s}$ and $k_{l}$ are constant thermal conductivities in the solid and liquid phases. 


\subsection{Heat transfer model in skin}

The temperature distribution in the skin region is determined by the Pennes' bio-heat equation $[2,8,9,10]$

$$
\rho c \frac{\partial T}{\partial t}=\frac{\partial}{\partial x}\left(k \frac{\partial T}{\partial x}\right)+\rho_{b} c_{b} \omega\left(T_{b}-T\right)+S \quad \text { in } R_{i} \text { for } i=4,5,6,
$$

where $\rho_{\mathrm{b}}$ and $c_{\mathrm{b}}$ are the density and specific heat of blood, $\omega$ is the rate of blood perfusion, $S$ is the rate of metabolic heat generation, and $T_{b}$ is the local arterial blood temperature. In general, $\mathrm{T}_{\mathrm{b}}$ is a function of the central blood pool temperature, the counter current heat exchange between arteries and veins, and body location [2]. Here we are unable to model the central blood pool temperature and the counter current heat exchange process so we assume $T_{b}$ is constant $[8,9]$.

In view of the properties of the skin and subcutaneous tissue, the thermal conductivities in each region are assumed constant but vary between layers [9] and the rate of metabolic heat generation and the rate of blood perfusion are negligible in the epidermis, uniform in subcutaneous tissue, and vary linearly in the dermis $[8,9]$. That is,

$$
\begin{aligned}
& \omega=S=0 \quad \text { in } R_{4}, \\
& \omega=\frac{x-x_{E}}{x_{D}-x_{E}} \omega_{0} \quad \text { and } \quad S=\frac{x-x_{E}}{x_{D}-x_{E}} s_{0} \quad \text { in } R_{5}, \\
& \omega=\omega_{0} \quad \text { and } S=s_{0} \quad \text { in } R_{6},
\end{aligned}
$$

where $x_{E}$ and $x_{D}$ denote the distance from the base of epidermis and dermis to the outer surface of the clothing respectively, $\omega_{0}$ and $s_{0}$ are maximum values of $\omega$ and $S$.

\subsection{Boundary and initial conditions}

Since a diver with the dry suit is immersed in water, radiant heat transfer is negligible at the outer surface. We assume Newton cooling at the water 
TABle 3: Parameters for the exterior boundary conditions.

\begin{tabular}{|cc|ccc|}
\hline Parameter & Value & Parameter & Water & Air \\
\hline $\mathrm{T}_{\mathrm{b}}\left[{ }^{\circ} \mathrm{C}\right]$ & $37^{\circ}$ & $\mathrm{P}\left[\mathrm{W} / \mathrm{m}^{2} /{ }^{\circ} \mathrm{K}\right]$ & 600 & 20 \\
$\mathrm{~h}_{\mathrm{b}}\left[\mathrm{W} / \mathrm{m}^{2} /{ }^{\circ} \mathrm{C}\right]$ & 65 & $\mathrm{~T}_{\mathrm{amb}}\left[{ }^{\circ} \mathrm{C}\right]$ & $4^{\circ}$ & $15^{\circ}$ \\
\hline
\end{tabular}

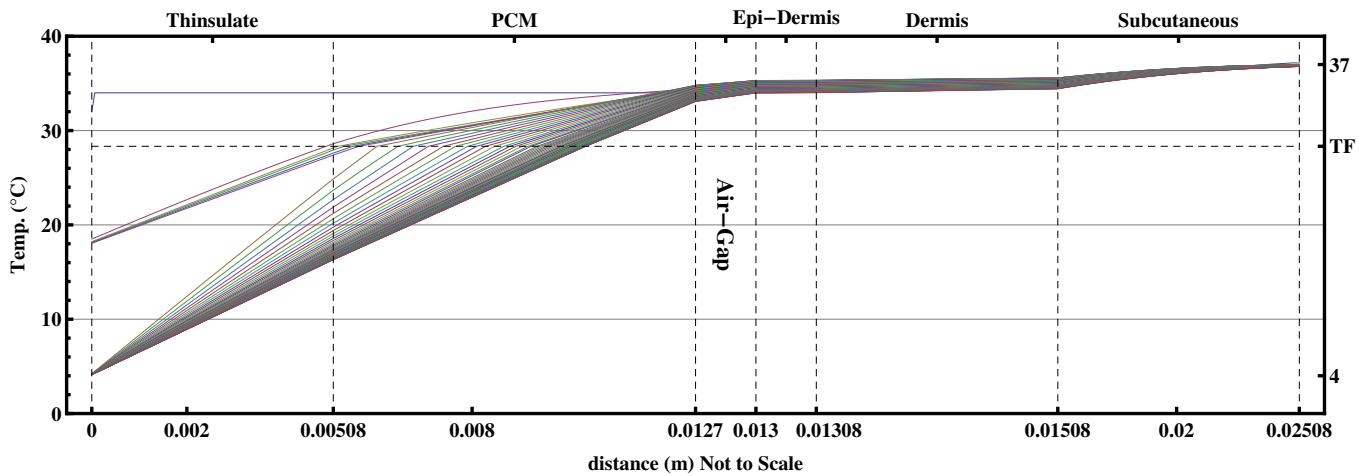

Figure 2: Temperature profiles plotted at one minute intervals for 65 minutes for the two-layered composite (air-gap $0.3 \mathrm{~mm}$ ). 
boundary $\Gamma_{1}$, then

$$
k(T) \frac{\partial T}{\partial x}(0, t)=P\left[T(0, t)-T_{a m b}\right] \text { on } \Gamma_{1}
$$

where $P$ is the surface heat transfer coefficient and $T_{a m b}$ is ambient temperature. During water immersion, the surface heat transfer coefficient $\mathrm{P}$ is many times greater than in air. Typical values of surface heat transfer coefficient, for icy water, range from 100 to $1000 \mathrm{~W} / \mathrm{m}^{2} /{ }^{\circ} \mathrm{K}$.

When analysing burn injury $[1,6]$, it is usual to assume the the temperature at the base of the subcutaneous layer is maintained at the body's core temperature of $37^{\circ} \mathrm{C}$. This is applicable for short time duration events like flash burns. To investigate the thermal properties of a dry suit immersed in icy water for up to 60 minutes, a boundary condition that is applicable to a wider range of environmental conditions is needed. At the base of subcutaneous layer we apply the boundary condition

$$
-k \frac{\partial T}{\partial x}=h_{b}\left(T-T_{b}\right) \text { on } \Gamma_{2},
$$

where $h_{b}$ is the heat transfer coefficient related to the convection process in the blood.

At all interior boundaries $\left(x=X_{T}, X_{P}, X_{A}, X_{E}, X_{D}\right)$, temperature and heat flux $(k \partial T / \partial x)$ are continuous.

Assuming that the body is initially at equilibrium with its surroundings, a steady state solution is determined for the skin layers with skin temperature set to $34^{\circ} \mathrm{C}$. The initial temperature in the suit and air layer is assumed to be constant at $34^{\circ} \mathrm{C}$. The diver in a dry suit then spends five minutes in air at $15^{\circ} \mathrm{C}(\mathrm{P}=20)$ before immersion in water at $4^{\circ} \mathrm{C}(\mathrm{P}=600)$ for 60 minutes. The parameters used in the numerical experiment are listed in Tables 1 to $3[1,3,6]$. 


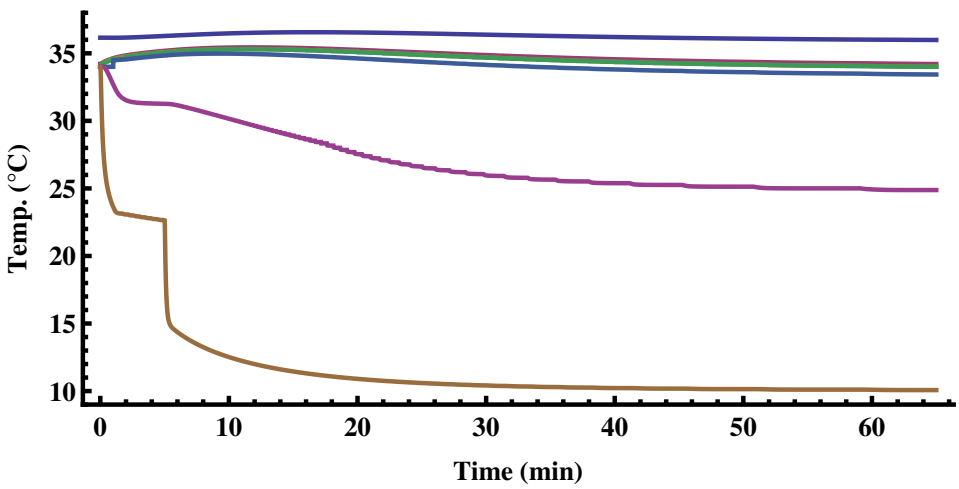

\section{- Subcutaneous \\ - Dermis \\ - Epidermis \\ - Skin Surface \\ - Air-gap \\ - PCM \\ - Thinsulate}

Figure 3: Temperature profiles at the skin boundary and in the middle of each layer against time for 40/60\% Thins./PCM composite (air-gap $0.3 \mathrm{~mm}$ ).

\subsection{Numerical method}

The governing equations, boundary conditions and initial conditions are integrated forward in time using finite differences. The Crank-Nicolson implicit scheme (unconditionally stable) is employed for Thinsulate, air-gap and skin layers. The thermal conductivity of air is very small relative to other regions and would impose a severe restriction of time step if an explicit scheme were employed.

The form of Equation (3) determines that an explicit finite difference scheme is applied to predict the temperature distribution in the PCM layer. We use a first order forward difference in time and conservative second order spatial differences [3]. The thermal conductivity $k(T)$, in the conservative scheme, is required at the mid-point of each grid cell. If the average temperature of adjacent grid points is greater than the fusion temperature, then $k(T)=k_{l}$ otherwise $k(T)=k_{s}$. This averaging of adjacent temperatures to determine the conductivity and the conservative scheme ensures that the enthalpy, temperature and the flux of heat are all continuous across the phase change interface (the temperature gradient is discontinuous). The averaging of 
adjacent grid points to determine the thermal conductivity is performed on all grid cells in the PCM layer. Hence no explicit knowledge of the position of the phase change interface is needed. Using the finite difference discretization of Equation (3) we integrate forward in time and determine the enthalpy (in the PCM layer) at the next time step. The temperature at the next time step is then determined uniquely from Equation (2).

The number of subintervals and grid spacing varies between each layer. This ensures that each layer contains appropriate numbers of nodal points to accurately determine temperature variation. In the Thinsulate and phase change layers 40 and 120 grid points are used. In the skin layers, (epidermis, dermis, subcutaneous), 4, 8 and 20 grid points are used. Two air-gap distances were considered, 0.3 and $1 \mathrm{~mm}$ with four and ten grid points used in each case. With 120 grid points in the PCM layer, stability of the explicit scheme requires the time step to be at most 0.01 secs. For 65 minutes 390, 000 time steps are performed. The large number of grid points in the PCM layer ensure a smoother solution where most of the variation occurs.

\section{$3 \quad$ Results and discussions}

The numerical simulations were implemented to evaluate the thermal performance of the diver dry suit fabricated with Thinsulate and PCM encapsulated in Thermosorb. Figure 2 (air-gap $0.3 \mathrm{~mm}$ ) shows the temperature distribution of composite fabric, air-gap, skin system at one minute intervals for five minutes while the diver with dry suit is in air of $15^{\circ} \mathrm{C}$ and for 60 minutes immersed in water at $4^{\circ} \mathrm{C}$. The horizontal axis shows the distance (not to scale) from the outer surface of the clothing. The temperature in the outer Thinsulate layer decreases rapidly. The decrease in temperature is delayed in the PCM layer due to the release of latent heat during solidification. The position of the phase change is indicated by the discontinuity in slope at the fusion temperature $\left(T_{f}\right)$. Initially the phase change moves rapidly through the PCM layer. As steady state is approached the phase change speed decreases to 


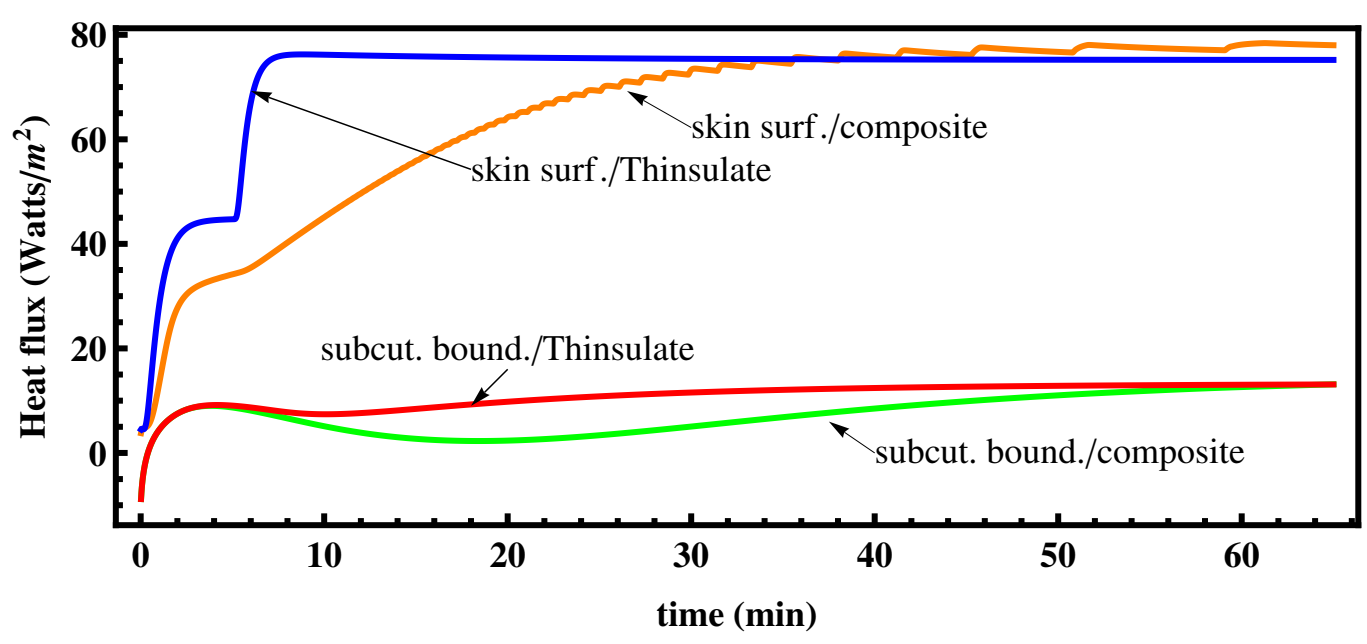

Figure 4: Heat flux at skin surface $\left(x=X_{A}\right)$ and subcutaneous boundary $\left(x=X_{S}\right)$ against time for $100 \%$ Thinsulate and $40 / 60 \%$ Thins./PCM composite (air-gap $0.3 \mathrm{~mm}$ ).

zero. Notice that not all the phase change material has changed from liquid to solid phase.

Figure 3 shows the temperature against time (for 65 minutes), at the mid-point of each layer and the skin surface when the air-gap is $0.3 \mathrm{~mm}$. The temperature in the skin layers initially increases. As the suit is applied heat loss from the skin surface decreases and the inflowing heat from blood profusion and metabolic production increase the temperature, ultimately decreasing the inflow of heat from blood profusion as the temperature difference between the blood and tissue decrease. After five minutes the change from air to water immersion can be seen in the sudden change in temperature in the Thinsulate layer. At steady state the temperature in each layer approaches a constant value.

Finally we compared a two-layer composite $(40 \%$ Thinsulate $+60 \%$ PCM $)$ with $100 \%$ Thinsulate of the same thickness. Figures 4 and 5 show the heat 
flux at skin surface $\left(x=X_{A}\right)$ and subcutaneous boundary $\left(x=X_{S}\right)$ against time for both fabrics when the air-gap is $0.3 \mathrm{~mm}$ and $1 \mathrm{~mm}$.

For the Thinsulate only dry suit (after immersion in water) the heat flux at the skin surface rapidly increases to its maximum. Within about two minutes heat is being lost at its maximum rate. Due to the heat stored in the PCM (as latent heat) the composite offers much greater thermal protection. Maximum heat loss is delayed for up to 25 minutes when the air-gap is $0.3 \mathrm{~mm}$ and for more than 30 minutes when the air-gap is $1 \mathrm{~mm}$. Note that maximum heat loss from the composite is greater because the PCM encapsulated in Thermosorb has a greater thermal conductance than Thinsulate. The stepping in the heat flux for the composite is due to the phase change moving across grid-cells. This can also be seen in the temperature at the mid-point of the PCM layer (see Figure 3). As the phase change moves across a cell the conductivity used in the conservative finite difference scheme changes from the liquid phase to solid phase value. This sudden change in conductivity causes the sudden jumps in temperature and heat flux which are more easily seen as the steady state is approached. Decreasing the grid spacing decreases the stepping distance without changing the overall results (still only a discrete approximation to the solution).

At the subcutaneous boundary $\left(x=X_{S}\right)$, when the suit is initially applied, the heat flux is negative as heat flows into the body core due to the decrease in heat loss from the skin surface and the excess production of heat from blood profusion and metabolism. Consequently as the temperature in the skin increases less heat perfuses from the arteries into the dermis and subcutaneous layer. As the heat flux from the skin surface increases, due to heat loss from the fabric surface in cool air, the temperature gradient in the skin increases and heat flows from the core into the skin and we see a positive heat flux at the subcutaneous boundary. As the temperature in the skin adjusts more heat perfuses from the arteries, less is required from the body core and the heat flux at the subcutaneous boundary decreases. After immersion in icy water, increased heat loss from the fabric surface causes an increased flow of heat from the skin surface. Eventually (about 5 mins for the Thinsulate only 


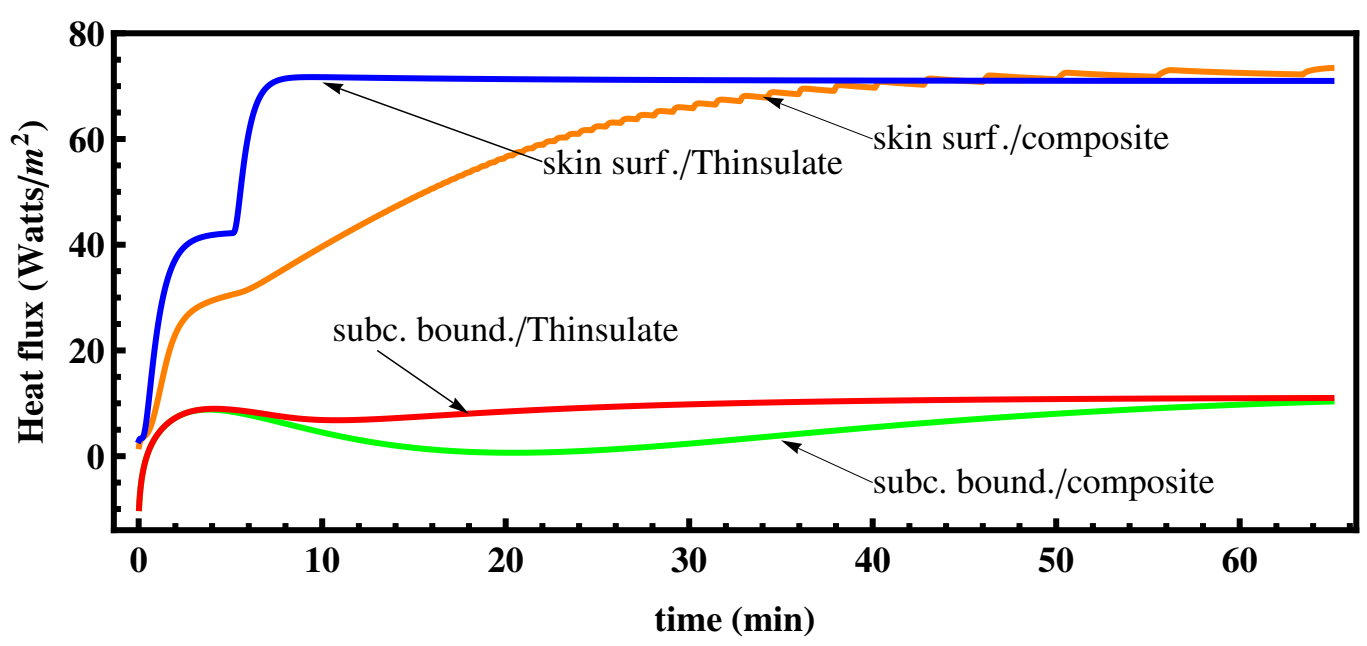

Figure 5: Heat flux at skin surface $\left(x=X_{A}\right)$ and subcutaneous boundary $\left(x=X_{S}\right)$ against time for $100 \%$ Thinsulate and $40 / 60 \%$ Thins./PCM composite (air-gap $1 \mathrm{~mm}$ ).

fabric) this increase in heat loss from the skin surface causes an increase in heat flow from the core into the skin. In the composite fabric the release of latent heat due to phase change causes a larger and more sustained decrease in heat flux at the subcutaneous boundary.

The numerical results demonstrate that the diver dry suit incorporating microencapsulated phase change material provides enhanced thermal protection under cold environmental conditions. Figures 4 and 5 show that the size of the air-gap, between suit and skin, is also an important factor in determining the thermal protection of the dry suit. 


\section{References}

[1] Chitrphiromsri, P., Kuznetsov, A. V., Modeling heat and moisture transport in firefighter protective clothing during flash fire exposure, J. Heat Mass Transfer., 41, 2005, 206-215. doi:10.1007/s00231-004-0504-x C627, C629, C632

[2] Dusan, F., Kevin, J.L. and Martin S., A computer model of human thermoregulation for a wide range of environmental conditions: the passive system, J Appl Physiol 87,1999, 1957-1972.

http://jap.physiology.org/cgi/content/abstract/87/5/1957 C630

[3] J. A. Gear, M. J. Lachut and Y. Ding. Enhanced thermal performance of garments embedded with encapsulated phase change material. ANZIAM J., 47(EMAC2005):C137-C151, 2006. http://anziamj . austms.org.au/ojs/index.php/ANZIAMJ/article/view/1035. C626, C629, C632, C633

[4] Ghali, K., Ghaddar, N., Harathani, J. and Jones, B., Experimental and numerical investigation of the effect of phase change materials on clothing during periodic ventilation, Textile Research Journal, 74(3), 2004, 205-214. doi:10.1177/004051750407400304 C626

[5] J. M. Hill and J. N. Dewynne. Heat Conduction. Applied Mathematics and Engineering Science Texts. Blackwell Scientific Publications, 1987. C628, C629

[6] Ng, E. Y. K., Tan, H. M. and Ooi, E. H., Boundary element method with bioheat equation for skin burn injury, J. Burns., 35, 2009, 987-997. doi:10.1016/j.burns.2009.01.010 C627, C629, C632

[7] Nuckols, M. L., Analytical modelling of a diver dry suit enhanced with micro-encapsulated phase change materials, J. Ocean Engineering., 26, 1999, 547-564. doi:10.1016/S0029-8018(98)00001-8 C626, C627, C628 
[8] Saxena, V. P. and Arya, D., Steady - state heat distribution in epidermis, dermis and subdermal tissues, J. Theor. Biol., 89, 1981, 423-432. doi:10.1016/0022-5193(81)90360-X C627, C630

[9] Saxena, V. P., Temperature distribution in human skin and subdermal tissues, J. theor. Biol., 102(2), 1983, 277-286.

doi:10.1016/0022-5193(83)90365-X C627, C630

[10] Wissler, E. H., Pennes' 1948 paper revisited., J. Appl. Physiol., 85, 1998, 35-41.

http://jap.physiology.org/cgi/content/abstract/85/1/35 C630

\section{Author addresses}

1. M. F. Ding, School of Mathematical and Geospatial Sciences, RMIT University, Melbourne, Australia. mailto:mingfang.ding@rmit.edu.au

2. J. A. Gear, School of Mathematical and Geospatial Sciences, RMIT University, Melbourne, Australia. mailto: jag@rmit.edu.au

3. Y. Ding, School of Mathematical and Geospatial Sciences, RMIT University, Melbourne, Australia. mailto:yan.ding@rmit.edu.au 